\title{
Finding and Counting Vertex-Colored Subtrees
}

\author{
Sylvain Guillemot ${ }^{1}$ and Florian Sikora ${ }^{2}$ \\ 1 Lehrstuhl für Bioinformatik, Friedrich-Schiller Universität Jena, Ernst-Abbe \\ Platz 2, 00743 Jena, Germany, E-mail: sylvain.guillemot@uni-jena.de \\ 2 Université Paris-Est, LIGM - UMR CNRS 8049, France \\ E-mail: sikora@univ-mlv.fr
}

\begin{abstract}
The problems studied in this article originate from the GRAPH MOTIF problem introduced by Lacroix et al. 17 in the context of biological networks. The problem is to decide if a vertex-colored graph has a connected subgraph whose colors equal a given multiset of colors $M$. Using an algebraic framework recently introduced by Koutis et al. [15] 16], we obtain new FPT algorithms for GRAPH MOTIF and variants, with improved running times. We also obtain results on the counting versions of this problem, showing that the counting problem is FPT if $M$ is a set, but becomes \#W[1]-hard if $M$ is a multiset with two colors.
\end{abstract}

\section{Introduction}

An emerging field in the modern biology is the study of the biological networks, which represent the interactions between biological elements [1]. A network is modeled by a vertex-colored graph, where nodes represent the biological compounds, edges represent their interactions, and colors represent functionalities of the graph nodes. Networks are often analyzed by studying their network motifs, which are defined as small recurring subnetworks. Motifs generally correspond to a set of elements realizing a same function, and which may have been evolutionarily preserved. Therefore, the discovery and the querying of motifs is a crucial problem [20], since it can help to decompose the network into functional modules, to identify conserved elements, and to transfer biological knowledge across species.

The initial definition of network motifs involves conservation of the topology and of the node labels; hence, looking for topological motifs is roughly equivalent to subgraph isomorphism, and thus is a computationally difficult problem. However, in some situations, the topology is not known or is irrelevant, which leads to searching for functional motifs instead of topological ones. In this setting, we still ask for the conservation of the node labels, but we replace topology conservation by the weaker requirement that the subnetwork should form a connected subgraph of the target graph. This approach was advocated by 17 and led to the definition of the GraPh Motif problem [10]: given a vertex-colored graph $G=(V, E)$ and a multiset of colors $M$, find a set $V^{\prime} \subseteq V$ such that the induced subgraph $G\left[V^{\prime}\right]$ is connected, and the multiset of colors of the vertices of $V^{\prime}$ is 
equal to $M$. In the literature, a distinction is made between the colorful case (when $M$ is a set), and the multiset case (when $M$ is an arbitrary multiset). Although this problem has been introduced for biological motivations, [3] points out that it may also be used in social or technical networks.

Not surprisingly, Graph Motif is NP-hard, even if $G$ is a bipartite graph with maximum degree 4 and $M$ is built over two colors only 10 . The problem is still NP-hard if $G$ is a tree, but in this case it can be solved in $\mathcal{O}\left(n^{2 c+2}\right)$ time, where $c$ is the number of distinct colors in $M$, while being $\mathrm{W}[1]$-hard for the parameter $c$ [10]. The difficulty of this problem is counterbalanced by its fixedparameter tractability when the parameter is $k$, the size of the solution [17,10,3]. The currently fastest FPT algorithms for the problem run in $\mathcal{O}^{*}\left(2^{k}\right)$ time for the colorful case, $\mathcal{O}^{*}\left(4.32^{k}\right)$ time for the multiset case, and use exponential space ${ }^{3}$.

Our contribution is twofold. First, we consider in Section 3 the decision versions of the GRAPH MOTIF problem, as well as some variants: we obtain improved FPT algorithms for these problems, by using the algebraic framework of multilinear detection for arithmetic circuits [15,16], presented in the next section. Second, we investigate in Section the counting versions of the GRAPH MotiF problem: instead of deciding if a motif appears in the graph, we now want to count the occurrences of this motif. This allows to assess if a motif is over- or under- represented in the network, by comparing the actual count of the motif to its expected count under a null hypothesis [19. We show that the counting problem is FPT in the colorful case, but becomes \#W[1]-hard for the multiset case with two colors. We refer the reader to [12,11] for definitions related to parameterized counting classes.

\section{Definitions}

This section contains definitions related to arithmetic circuits, and to the MULTILinear Detection (MLD) problem. It concludes by stating Theorem $\mathbb{1}$, which will be used throughout the paper.

\subsection{Arithmetic circuits}

In the following, a capital letter $X$ will denote a set of variables, and a lowercase letter $x$ will denote a single variable. If $X$ is a set of variables and $\mathbb{A}$ is a commutative ring, we denote by $\mathbb{A}[X]$ the ring of multivariate polynomials with coefficients in $\mathbb{A}$ and involving variables of $X$. Given a monomial $m=x_{1} \ldots x_{k}$ in $\mathbb{A}[X]$, where the $x_{i}$ s are variables, its degree is $k$, and $m$ is multilinear iff its variables are distinct.

An arithmetic circuit over $X$ is a pair $\mathcal{C}=(C, r)$, where $C$ is a labeled directed acyclic graph (dag) such that (i) the children of each node are totally ordered, (ii) the nodes are labeled either by op $\in\{+, \times\}$ or by an element of $X$, (iii) no

\footnotetext{
${ }^{3}$ We use the notations $\mathcal{O}^{*}$ and $\tilde{\mathcal{O}}$ to suppress polynomial and polylogarithmic factors, respectively.
} 
internal node is labeled by an element of $X$, and where $r$ is a distinguished node of $C$ called the root. We denote by $V_{\mathcal{C}}$ the set of nodes of $C$, and for a given node $u$ we denote by $N_{C}(u)$ the set of children (i.e. out-neighbors) of $u$ in $C$. We recall that a node $u$ is called a leaf of $C$ iff $N_{C}(u)=\emptyset$, an internal node otherwise. We denote by $T(\mathcal{C})$ the size of $\mathcal{C}$ (defined as the number of arcs), and we denote by $S(\mathcal{C})$ the number of nodes of $\mathcal{C}$ of indegree $\geq 2$.

Given a commutative ring $\mathbb{A}$, evaluating $\mathcal{C}$ over $\mathbb{A}$ under a mapping $\phi: X \rightarrow \mathbb{A}$ consists in computing, for each node $u$ of $C$, a value $\operatorname{val}(u) \in \mathbb{A}$ as follows: 1 . for a leaf $u$ labeled by $x \in X$, we let $\operatorname{val}(u)=\phi(x), 2$. for an internal node $u$ labeled by $+($ resp. $\times)$, we compute $\operatorname{val}(u)$ as the sum (resp. product) of the values of its children. The result of the evaluation is then $\operatorname{val}(r)$. The symbolic evaluation of $\mathcal{C}$ is the polynomial $P_{\mathcal{C}} \in \mathbb{Z}[X]$ obtained by evaluating $\mathcal{C}$ over $\mathbb{Z}[X]$ under the identity mapping $\phi: X \rightarrow \mathbb{Z}[X]$.

We stress that the above definition of arithmetic circuits does not allow constants, a restriction which is necessary for the algorithms. However, we can safely allow the two constants $0_{\mathbb{A}}$ and $1_{\mathbb{A}}$, the zero and the unit of $\mathbb{A}$ (which is assumed to be a unital ring). For simplicity, these two constants will be represented by an empty sum and an empty product, respectively.

\subsection{Multilinear Detection}

Informally, the Multilinear Detection problem asks, for a given arithmetic circuit $\mathcal{C}$ and an integer $k$, if the polynomial $P_{\mathcal{C}}$ has a multilinear monomial of degree $k$. However, this definition does not give a certificate checkable in polynomial-time, so for technical reasons we define the problem differently.

A monomial-subtree of $\mathcal{C}$ is a pair $T=\left(\mathcal{C}^{\prime}, \phi\right)$, where $\mathcal{C}^{\prime}=\left(C^{\prime}, r^{\prime}\right)$ is an arithmetic circuit over $X$ whose underlying dag $C^{\prime}$ is a directed tree, and where $\phi: V_{\mathcal{C}^{\prime}} \rightarrow V_{\mathcal{C}}$ is such that (i) $\phi\left(r^{\prime}\right)=r$, (ii) if $u \in V_{\mathcal{C}^{\prime}}$ is labeled by $x \in X$, then so is $\phi(u)$, (iii) if $u \in V_{\mathcal{C}^{\prime}}$ is labeled by + then so is $\phi(u)$, and $N_{\mathcal{C}^{\prime}}(u)$ consists of a single element $v \in N_{\mathcal{C}}(\phi(u))$, (iv) if $u \in V_{\mathcal{C}^{\prime}}$ is labeled by $\times$, then so is $\phi(u)$, and $\phi$ maps bijectively $N_{\mathcal{C}^{\prime}}(u)$ into $N_{\mathcal{C}}(\phi(u))$ by preserving the ordering on siblings. The variables of $T$ are the leaves of $C^{\prime}$ labeled by variables in $X$. We say that $T$ is distinctly-labeled iff its variables are distinct.

Intuitively, a monomial-subtree tells us how to construct a multilinear from the circuit: Condition (i) tells us to start at the root, Condition (iii) tells us that when reaching a + node we are only allowed to pick one child, and Condition (iv) tells us that when reaching a $\times$ node we have to pick all children. The (distinctly-labeled) monomial-subtrees of $\mathcal{C}$ with $k$ variables will then correspond to the (multilinear) monomials of $P_{\mathcal{C}}$ having degree $k$. Therefore, we formulate the Multilinear Detection problem as follows:

Name: Multilinear Detection (MLD)

Input: An arithmetic circuit $\mathcal{C}$ over a set of variables $X$, an integer $k$

Solution: A distinctly-labeled monomial-subtree of $\mathcal{C}$ with $k$ variables. 
Solving MLD amounts to decide if $P_{\mathcal{C}}$ has a multilinear monomial of degree $k$ (observe that there are no possible cancellations), and solving \#MLD amounts to compute the sum of the coefficients of multilinear monomials of $P_{\mathcal{C}}$ having degree $k$. The restriction of MLD when $|X|=k$ is called ExACT Multilinear DETECTION (XMLD). In this article, we will rely on the following far-reaching result from [21, 16] to obtain new algorithms for GRAPH MOTIF:

Theorem 1 ([21, 16]). MLD can be solved by a randomized algorithm which uses $\tilde{\mathcal{O}}\left(2^{k} T(\mathcal{C})\right)$ time and $\tilde{\mathcal{O}}(S(\mathcal{C}))$ space.

\section{$3 \quad$ Finding vertex-colored subtrees}

In this section, we consider several variants of the GRAPH MOTIF problem, and we obtain improved FPT algorithms for these problems by reduction to MLD. Notably, we obtain $\mathcal{O}^{*}\left(2^{k}\right)$ time algorithms for problems involving colorful motifs, and $\mathcal{O}^{*}\left(4^{k}\right)$ time algorithms for multiset motifs.

\subsection{The colorful case}

In the colorful formulation of the problem, the graph is vertex-colored, and we seek a subtree with $k$ vertices having distinct colors. This leads to the following formal definition:

Name: Colorful Graph Motif (CGM)

Input: A graph $G=(V, E), k \in \mathbb{N}$, a set $C$, a function $\chi: V \rightarrow C$

Solution: A subtree $T=\left(V_{T}, E_{T}\right)$ of $G$ s.t. (i) $\left|V_{T}\right|=k$ and (ii) for each $u, v \in V_{T}$ distinct, $\chi(u) \neq \chi(v)$.

The restriction of Colorful Graph Motif when $|C|=k$ is called ExACT Colorful Graph Motif (XCGM). Note that this restriction requires that the vertices of $T$ are bijectively labeled by the colors of $C$. In [7], the XCGM problem was shown to be solvable in $\mathcal{O}^{*}\left(2^{k}\right)$ time and space, while it is not difficult to see that the general CGM problem can be solved in $\mathcal{O}^{*}\left((2 e)^{k}\right)$ time and $\mathcal{O}^{*}\left(2^{k}\right)$ space by color-coding. By using a reduction to Multilinear Detection, we improve upon these complexities. In the following, we let $n$ and $m$ denote the number of vertices and the number of edges of $G$, respectively.

Proposition 1. CGM is solvable by a randomized algorithm in $\tilde{\mathcal{O}}\left(2^{k} k^{2} m\right)$ time and $\tilde{\mathcal{O}}(k n)$ space.

Proof (Sketch). Let $I$ be an instance of CGM. We construct the following circuit $\mathcal{C}_{I}$ : its set of variables is $\left\{x_{c}: c \in C\right\}$, and we introduce intermediary nodes $P_{i, u}$ for $1 \leq i \leq k, u \in V$, as well as a root node $P$. Informally, the multilinear monomials of $P_{i, u}$ will correspond to colorful subtrees of $G$ having $i$ vertices, 
including $u$. The definitions are as follows:

$$
P_{i, u}=\sum_{i^{\prime}=1}^{i-1} \sum_{v \in N_{G}(u)} P_{i^{\prime}, u} P_{i-i^{\prime}, v} \text { if } i>1, \quad P_{1, u}=x_{\chi(u)}
$$

and $P=\sum_{u \in V} P_{k, u}$. The resulting instance of MLD is $I^{\prime}=\left(\mathcal{C}_{I}, k\right)$. By applying Theorem 11, and by observing that $T\left(\mathcal{C}_{I}\right)=\mathcal{O}\left(k^{2} m\right)$ and $S\left(\mathcal{C}_{I}\right)=\mathcal{O}(k n)$, we solve $I^{\prime}$ in $\tilde{\mathcal{O}}\left(2^{k} k^{2} m\right)$ time and $\tilde{\mathcal{O}}(k n)$ space. The correctness of the construction follows by showing by induction on $1 \leq i \leq k$ that: $x_{c_{1}} \ldots x_{c_{d}}$ is a multilinear monomial of $P_{i, u}$ iff (i) $d=i$ and (ii) there exists $T=\left(V_{T}, E_{T}\right)$ colorful subtree of $G$ such that $u \in V_{T}$ and $\chi\left(V_{T}\right)=\left\{c_{1}, \ldots, c_{d}\right\}$.

\subsection{The multiset case}

We consider the multiset formulation of the problem: we now allow some colors to be repeated but impose a maximum number of occurrences for each color. This problem can be seen as a generalization of the original GraPH MotiF problem.

Given a multiset $M$ over a set $A$, and given an element $x \in A$, we denote by $n_{M}(x)$ the number of occurrences of $x$ in $M$. Given two multisets $M, M^{\prime}$, we denote their inclusion by $M \subseteq M^{\prime}$. We denote by $|M|$ the size of $M$, where elements are counted with their multiplicities. Given two sets $A, B$, a function $f: A \rightarrow B$ and a multiset $X$ over $A$, we let $f(X)$ denote the multiset containing the elements $f(x)$ for $x \in X$, counted with multiplicities; precisely, given $y \in B$ we have $n_{f(X)}(y)=\sum_{x \in A: f(x)=y} n_{X}(x)$.

We now define the following two variants of Colorful Graph Motif, which allow for multiset motifs:

Name: Multiset Graph Motif (MGM)

Input: A graph $G=(V, E)$, an integer $k$, a set $C$, a function $\chi: V \rightarrow C$, a multiset $M$ over $C$.

Solution: A subtree $T=\left(V_{T}, E_{T}\right)$ of $G$ s.t. (i) $\left|V_{T}\right|=k$ and (ii) $\chi\left(V_{T}\right) \subseteq M$.

Name: Multiset Graph Motif With Gaps (MGMG)

Input: A graph $G=(V, E)$, integers $k, r$, a set $C$, a function $\chi: V \rightarrow C$, a multiset $M$ over $C$.

Solution: A subtree $T=\left(V_{T}, E_{T}\right)$ of $G$ s.t. (i) $\left|V_{T}\right| \leq r$ and (ii) there exists $S \subseteq V_{T}$ of size $k$ such that $\chi(S) \subseteq M$.

The restriction of Multiset Graph Motif when $|M|=k$ is called Exact Multiset Graph Motif (XMGM). Note that in this case we require that $T$ contains every occurrence of $M$, i.e. $\chi\left(V_{T}\right)=M$. In this way, the XMGM problem coincides with the GRAPH Motif problem defined in [10,3], while the MGM problem is the parameterized version of the MAX MoTIF problem considered in [9]. The notion of gaps is introduced in [17], and encompasses the notion of insertions and deletions of [7]. 
Previous algorithms for these problems relied on color-coding [2]; these algorithms usually have an exponential space complexity, and a high time complexity. For the GRAPH Motif problem, [10] gives a randomized algorithm with an implicit $\mathcal{O}\left(87^{k} \mathrm{~km}\right)$ running time, while [3] describes a first randomized algorithm running in $\mathcal{O}\left(8.16^{k} m\right)$, and shows a second algorithm with $\mathcal{O}\left(4.32^{k} k^{2} m\right)$ running time, using two different speed-up techniques (清 and [13). For the MAx MoTIF problem, [9] presents a randomized algorithm with an implicit $\mathcal{O}\left(\left(32 e^{2}\right)^{k} k m\right)$ running time. Here again, we can apply Theorem 1 to improve the time and space complexities:

Proposition 2. 1. MGM is solvable by a randomized algorithm in $\tilde{\mathcal{O}}\left(4^{k} k^{2} m\right)$ time and $\tilde{\mathcal{O}}(k n)$ space.

2. MGMG is solvable by a randomized algorithm in $\tilde{\mathcal{O}}\left(4^{k} r^{2} m\right)$ time and $\tilde{\mathcal{O}}(r n)$ space.

Proof. Point 1. We modify the circuit of Proposition 1 as follows. For each color $c \in C$ with $n_{M}(c)=m$, we introduce variables $y_{c, 1}, \ldots, y_{c, m}$, and we introduce a node $Q_{c}=y_{c, 1}+\ldots+y_{c, m}$. For each vertex $u \in V$, we introduce a variable $x_{u}$, and we define:

$$
P_{i, u}=\sum_{i^{\prime}=1}^{i-1} \sum_{v \in N_{G}(u)} P_{i^{\prime}, u} P_{i-i^{\prime}, v} \text { if } i>1, \quad P_{1, u}=x_{u} Q_{\chi(u)}
$$

and $P=\sum_{u \in K} P_{k, u}$. Note that we changed only the base case in the recurrence of Proposition 1. The intuition is that the variables $x_{u}$ will ensure that we choose different vertices to construct the tree, and that the variables $y_{c, i}$ will ensure that a given color cannot occur more than required. The resulting instance of MLD is $I^{\prime}=\left(\mathcal{C}_{I}, 2 k\right)$, and since $T\left(\mathcal{C}_{I}\right)=\mathcal{O}\left(k^{2} m\right)$ and $S\left(\mathcal{C}_{I}\right)=\mathcal{O}(k n)$, we solve it in the claimed bounds by Theorem 1. A similar induction as in Proposition 11 shows that: for every $1 \leq i \leq k$, a multilinear monomial of $P_{i, u}$ has the form $x_{v_{1}} y_{c_{1}, j_{1}} \ldots x_{v_{i}} y_{c_{i}, j_{i}}$, and it is present iff there is a subtree $\left(V_{T}, E_{T}\right)$ of $G$ such that $u \in V_{T}, V_{T}=\left\{v_{1}, \ldots, v_{i}\right\}$ and $\chi\left(V_{T}\right)=\left\{\left\{c_{1}, \ldots, c_{i}\right\} \subseteq M\right.$.

Point 2. We modify the construction of Point 1 by now setting $P_{1, u}=1+$ $x_{u} Q_{\chi(u)}$ for each $u \in V$, and $P=\sum_{u \in V} \sum_{i=1}^{r} P_{i, u}$. Informally, adding the constant 1 to each $P_{1, u}$ permits to ignore some vertices of the subtree, allowing to only select a set $S$ of $k$ vertices such that $\chi(S) \subseteq M$. The correctness of the construction is shown by a similar induction as above. The catch here is that when considering two trees $T_{1}, T_{2}$ obtained from $P_{i^{\prime}, u}, P_{i-i^{\prime}, v}$, their selected vertices will be distinct, but they may have "ignored" vertices in common; we can then find a subset of $E\left(T_{1}\right) \cup E\left(T_{2}\right) \cup\{u v\}$ which forms a tree containing all selected vertices from $T_{1}, T_{2}$.

\subsection{Edge-weighted versions}

We consider an edge-weighted variant of the problem, where the subtree is now required to have a given total weight, in addition to respecting the color constraints. This variant has been studied in [6] under the name EDGE-WEIGHTED 
Graph Motif. In our case, we define two problems, depending on whether we consider colorful or multiset motifs.

Name: Weighted Colorful Graph Motif (WCGM)

Input: A complete graph $G=(V, E)$, a function $\chi: V \rightarrow C$, a weight function $w: E \rightarrow \mathbb{N}$, integers $k, r$

Solution: A subtree $T=\left(V_{T}, E_{T}\right)$ of $G$ such that (i) $\left|V_{T}\right|=k$, (ii) $\chi$ is injective on $V_{T}$, (iii) $\sum_{e \in E_{T}} w(e) \leq r$.

Name: Weighted Multiset Graph Motif (WMGM)

Input: A complete graph $G=(V, E)$, a function $\chi: V \rightarrow C$, a weight function $w: E \rightarrow \mathbb{N}$, integers $k, r$, a multiset $M$

Solution: A subtree $T=\left(V_{T}, E_{T}\right)$ of $G$ such that (i) $\left|V_{T}\right|=k$, (ii) $\chi\left(V_{T}\right) \subseteq M$, (iii) $\sum_{e \in E_{T}} w(e) \leq r$.

We observe that the WMGM problem contains as a special case the MiN$\mathrm{CC}$ problem introduced in [8], which seeks a subgraph respecting the multiset motif, and having at most $r$ connected components. Indeed, we can easily reduce Min-CC to WMGM: given the graph $G$, we construct a complete graph $G^{\prime}$ with the same vertex set, and we assign a weight 0 to edges of $G$, and a weight 1 to non-edges of $G$.

Proposition 3. 1. WCGM is solvable by a randomized algorithm in $\tilde{\mathcal{O}}\left(2^{k} k^{2} r^{2} m\right)$ time and $\tilde{\mathcal{O}}(k r n)$ space.

2. WMGM is solvable by a randomized algorithm in $\tilde{\mathcal{O}}\left(4^{k} k^{2} r^{2} m\right)$ time and $\tilde{\mathcal{O}}(k r n)$ space.

Proof. We only prove 1, since 2 relies on the same modification as in Proposition 2. The construction of the arithmetic circuit is similar to the construction in Proposition 1. The set of variables is $\left\{x_{c}: c \in C\right\}$, and we introduce nodes $P_{i, j, u}$, for $1 \leq i \leq k$ and $0 \leq j \leq r$, whose multilinear monomials will correspond to colorful subtrees having $i$ vertices including $u$, and with total weight $\leq j$. The definitions are as follows:

$$
\begin{aligned}
P_{1, j, u} & =x_{\chi(u)} \\
P_{i, j, u} & =\sum_{i^{\prime}=1}^{i-1} \sum_{v \in V} \sum_{j^{\prime}=0}^{j-w(u v)} P_{i^{\prime}, j^{\prime}, u} P_{i-i^{\prime}, j-j^{\prime}-w(u v), v} \text { if } i>1
\end{aligned}
$$

and $P=\sum_{u \in V} P_{k, r, u}$. The resulting instance of MLD is $I^{\prime}=\left(\mathcal{C}_{I}, k\right)$, and since $T\left(\mathcal{C}_{I}\right)=\mathcal{O}\left(k^{2} r^{2} m\right)$ and $S\left(\mathcal{C}_{I}\right)=\mathcal{O}(k r n)$, we solve it in the claimed bounds by Theorem 1 . The correctness of the construction follows by showing that: given $1 \leq i \leq k, 0 \leq j \leq r, u \in V, x_{c_{1}} \ldots x_{c_{d}}$ is a multilinear monomial of $P_{i, j, u}$ iff (i) $d=i$ and (ii) there exists $T=\left(V_{T}, E_{T}\right)$ colorful subtree of $G$ with $u \in V_{T}, \chi\left(V_{T}\right)=\left\{c_{1}, \ldots, c_{d}\right\}$ and $\sum_{e \in E_{T}} w(e) \leq j$. 


\section{Counting vertex-colored subtrees}

In this section, we consider the counting versions of the problems XCGM and XMGM introduced in Section 3. For the former, we show that its counting version \#XCGM is FPT; for the latter, we prove that its counting version \#XMGM is \#W[1]-hard.

\subsection{FPT algorithms for the colorful case}

We show that \#XCGM is fixed-parameter tractable (Proposition 5). We rely on a general result for \#XMLD (Proposition 4 ), which uses inclusion-exclusion as in 14].

Say that a circuit $\mathcal{C}$ is $k$-bounded iff $P_{\mathcal{C}}$ has only monomials of degree $\leq k$. Observe that given a circuit $\mathcal{C}$, we can efficiently transform it in a $k$-bounded circuit $\mathcal{C}^{\prime}$ such that (i) $\mathcal{C}$ and $\mathcal{C}^{\prime}$ have the same monomials of degree $k$, (ii) $\left|\mathcal{C}^{\prime}\right| \leq(k+1)^{2}|\mathcal{C}|$; the details of the construction are omitted 1 . The following result shows that we can efficiently count solutions for $k$-bounded circuits with $k$ variables (and thus for general circuits, with an extra $\mathcal{O}\left(k^{2}\right)$ factor in the complexity).

Proposition 4. \#XMLD for $k$-bounded circuits is solvable in $\mathcal{O}\left(2^{k} T(\mathcal{C})\right)$ time and $\mathcal{O}(S(\mathcal{C}))$ space.

Proof. Let $\mathcal{C}$ be the input circuit on a set $X$ of $k$ variables. For a monomial $m$ let $\operatorname{Var}(m)$ denote its set of variables. Given $S \subseteq X$, let $N_{S}$, resp. $N_{S}^{\prime}$, be the number of monomials $m$ of $P_{\mathcal{C}}$ such that $\operatorname{Var}(m)=S$, resp. $\operatorname{Var}(m) \subseteq S$. Observe that for every $S \subseteq X$, we have $N_{S}^{\prime}=\sum_{T \subseteq S} N_{T}$. Therefore, by Möbius inversion it holds that for every $S \subseteq X, N_{S}=\sum_{T \subseteq S}(-1)^{|S \backslash T|} N_{T}^{\prime}$.

Since $\mathcal{C}$ is $k$-bounded, $N_{X}$ is the number of multilinear monomials of $P_{\mathcal{C}}$ having degree $k$. Now, each value $N_{S}^{\prime}$ can be computed by evaluating $\mathcal{C}$ under the mapping $\phi: X \rightarrow \mathbb{Z}$ defined by $\phi(v)=1$ if $v \in S, \phi(v)=0$ if $v \notin S$. By the Möbius inversion formula, we can thus compute the desired value $N_{X}$ in $\mathcal{O}\left(2^{k} T(\mathcal{C})\right)$ time and $\mathcal{O}(S(\mathcal{C}))$ space.

It is worth mentioning that Proposition 1 generalizes several counting algorithms based on inclusion-exclusion, such as the well-known algorithm for \#HAmiltonian Path of [14], as well as results of 18. Indeed, the problems considered in these articles can be reduced to counting multilinear monomials of degree $n$ for circuits with $n$ variables (where $n$ is usually the number of vertices of the graph), which leads to algorithms running in $\mathcal{O}^{*}\left(2^{n}\right)$ time and polynomial space.

Let us now turn to applying Proposition to the \#XCGM problem. Recall that we defined in Proposition 1 a circuit $\mathcal{C}_{I}$ for the general CGM problem; we will have to modify it slightly for the purpose of counting solutions.

\footnotetext{
${ }^{4}$ The idea is to assume w.l.o.g. that $\mathcal{C}$ has outdegree 2 . Then, we create $k+1$ copies $u_{0}, \ldots, u_{k}$ of each node $u$ of $\mathcal{C}$, such that the monomials of $u_{i}$ correspond to the degree $i$-monomials of $u$. If $r$ is the root node of $\mathcal{C}$, then $r_{k}$ becomes the root node of $\mathcal{C}^{\prime}$.
} 
Proposition 5. \#XCGM is solvable in $\mathcal{O}\left(2^{k} k^{3} m\right)$ time and $\mathcal{O}\left(k^{2} n\right)$ space.

Proof. Let $I$ be an instance of XCGM. A rooted solution for $I$ is a pair $(u, T)$ where $T$ is a solution of XCGM on $I$ and $u$ is a vertex of $T$ (which should be seen as the root of the tree). The solutions of XCGM on $I$ are also called unrooted solutions. Let $N_{r}(I)$ and $N_{u}(I)$ be the number of rooted, resp. unrooted, solutions for $I$. We will show how to compute $N_{r}(I)$ in the claimed time and space bounds; since $N_{u}(I)=\frac{N_{r}(I)}{k}$, the result will follow.

To compute $N_{r}$, observe first that we cannot apply Proposition 4 to the circuit $\mathcal{C}_{I}$ of Proposition 1. Indeed, the circuit $\mathcal{C}_{I}$ counts the ordered subtrees, and not the unordered ones. Therefore, we need to modify the circuit in the following way: at each vertex $v$ of $V_{T}$, we examine its children by increasing color. This leads us to define the following circuit $\mathcal{C}_{I}^{\prime}$ : suppose w.l.o.g. that $C=\{1, \ldots, k\}$, introduce nodes $P_{i, j, u}$ for each $1 \leq i \leq k, 1 \leq j \leq k+1, u \in V$, variables $x_{i}$ for each $1 \leq i \leq k$, and define:

$$
\begin{aligned}
& P_{1, j, u}=x_{\chi(u)}, \quad P_{i, j, u}=0 \text { if } i \geq 2, j=k+1 \\
& P_{i, j, u}=P_{i, j+1, u}+\sum_{i^{\prime}=1}^{i-1} \sum_{v \in N_{G}(u): \chi(v)=j} P_{i^{\prime}, j+1, u} P_{i-i^{\prime}, 1, v} \text { if } i \geq 2,1 \leq j \leq k
\end{aligned}
$$

Let us also introduce a root node $P=\sum_{u \in V} P_{k, 1, u}$. Given $1 \leq i, j \leq k$ and $u \in V$, let $\mathcal{S}_{i, j, u}$ denote the set of pairs $(u, T)$ where (i) $T$ is a properly colored subtree of $I$ containing $u$ and having $i$ vertices, (ii) the neighbors of $u$ in $T$ have colors $\geq j$. It can be shown by induction on $i$ that: there is a bijection between $\mathcal{S}_{i, j, u}$ and the multilinear monomials of $P_{i, j, u}$. Therefore, the number of multilinear monomials of $P$ is equal to $N_{r}$; since $T\left(\mathcal{C}_{I}^{\prime}\right)=\mathcal{O}\left(k^{3} m\right), S\left(\mathcal{C}_{I}^{\prime}\right)=$ $\mathcal{O}\left(k^{2} n\right)$ and since $\mathcal{C}_{I}^{\prime}$ is $k$-bounded, it follows by Proposition 1 that $N_{r}$ can be computed in $\mathcal{O}\left(2^{k} k^{3} m\right)$ time and $\mathcal{O}\left(k^{2} n\right)$ space.

\subsection{Hardness of the multiset case}

In this subsection, we show that \#XMGM is \#W[1]-hard. For convenience, we first restate the problem in terms of vertex-distinct embedded subtrees.

Let $G=(V, E)$ and $H=\left(V^{\prime}, E^{\prime}\right)$ be two multigraphs. An homomorphism of $G$ into $H$ is a pair $\phi=\left(\phi_{V}, \phi_{E}\right)$ where $\phi_{V}: V \rightarrow V^{\prime}$ and $\phi_{E}: E \rightarrow E^{\prime}$, such that if $e \in E$ has endpoints $x, y$ then $\phi_{E}(e)$ has endpoints $\phi_{V}(x), \phi_{V}(y)$. An embedded subtree of $G$ is denoted by $\mathcal{T}=\left(T, \phi_{V}, \phi_{E}\right)$ where $T=\left(V_{T}, E_{T}\right)$ is a tree, and $\left(\phi_{V}, \phi_{E}\right)$ is an homomorphism from $T$ into $G$. We say that $\mathcal{T}$ is a vertex-distinct embedded subtree of $G$ (a "vdst" of $G$ ) if $\phi_{V}$ is injective. We say $\mathcal{T}$ is an edge-distinct embedded subtree of $G$ (an "edst" of $G$ ) iff $\phi_{E}$ is injective. We restate XMGM as follows:

Name: Exact Multiset Graph Motif (XMGM)

Input: A graph $G=(V, E)$, an integer $k$, a set $C$, a function $\chi: V \rightarrow C$, a multiset $M$ over $C$ s.t. $|M|=k$. 
Solution: A vdst $\left(T, \phi_{V}, \phi_{E}\right)$ of $G$ s.t. $\chi \circ \phi_{V}\left(V_{T}\right)=M$.

We first show the hardness of two intermediate problems (Lemma 1). Before defining these problems, we need the following notions. Consider a multigraph $G=(V, E)$. Consider a partition $\mathcal{P}$ of $V$ into $V_{1}, \ldots, V_{k}$, and a tuple $t \in[r]^{k}$. A $(\mathcal{P}, t)$-mapping from a set $A$ is an injection $\psi: A \rightarrow V \times[r]$ such that for every $x \in A$, if $\psi(x)=(v, i)$ with $v \in V_{j}$, then $1 \leq i \leq t_{j}$. From $\psi$, we define its reduction as the function $\psi^{r}: A \rightarrow V$ defined by $\psi^{r}(x)=v$ whenever $\psi(x)=(v, i)$. We also define a tuple $T(\psi)=\left(n_{1}, \ldots, n_{k}\right) \in[r]^{k}$ such that for each $i \in[k], n_{i}=\max _{v \in V_{i}}\left|\left\{x \in A: \psi^{r}(x)=v\right\}\right|$.

Given two tuples $t, t^{\prime} \in[r]^{k}$, denote $t \leq t^{\prime}$ iff $t_{i} \leq t_{i}^{\prime}$ for each $i \in[k]$. Note that for a $(\mathcal{P}, t)$-mapping $\psi$, we always have $T(\psi) \leq t$ since $\psi$ is injective. We say that a $(\mathcal{P}, t)$-labeled edst for $G$ is a tuple $\left(T, \psi_{V}, \psi_{E}\right)$ where (i) $T=\left(V_{T}, E_{T}\right)$ is a tree, (ii) $\psi_{V}$ is a $(\mathcal{P}, t)$-mapping from $V_{T}$, (iii) $\left(T, \psi_{V}^{r}, \psi_{E}\right)$ is an edst of $G$. Our intermediate problems are defined as follows:

Name: Multicolored Embedded Subtree-1 (MEST - 1)

Input: Integers $k, r$, a $k$-partite multigraph $G$ with partition $\mathcal{P}$, a tuple $t \in[r]^{k}$ Solution: A $(\mathcal{P}, t)$-labeled edst $\left(T, \psi_{V}, \psi_{E}\right)$ for $G$ s.t. $\left|V_{T}\right|=r$ and $T\left(\psi_{V}\right)=t$.

The MEST - 2 problem is defined similarly, except that we do not require that $T\left(\psi_{V}\right)=t$ (and thus we only have $T\left(\psi_{V}\right) \leq t$ ). While we will only need \#MEST -2 in our reduction for \#XMGM, we first show the hardness of \#MEST -1 , then reduce it to \#MEST -2 .

Lemma 1. \#MEST - 1 and \#MEST - 2 are \#W[1]-hard for parameter $(k, r)$.

The proof is omitted due to space constraints.

Proposition 6. \#XMGM is \#W[1]-hard for parameter $k$.

Proof. We reduce from \#MEST -2 , and conclude using Lemma 1. Let $I=$ $(k, r, G, t)$ be an instance of \#MEST -2 , where $G=(V, E)$ is a multigraph, and let $\mathcal{S}_{I}$ be its set of solutions. From $G$, we construct a graph $H$ as follows: (i) we subdivide each edge $e \in E$, creating a new vertex $a[e]$, (ii) we substitute each vertex $v \in V_{i}$ by an independent set formed by $t_{i}$ vertices $b[v, 1], \ldots, b\left[v, t_{i}\right]$. We let $A$ be the set of vertices $a[e]$ and $B$ the set of vertices $b[v, i]$, we therefore have a bipartite graph $H=(A \cup B, F)$. We let $I^{\prime}=(H, 2 r-1, C, \chi, M)$, where $C=\{1,2\}, \chi$ maps $A$ to 1 and $B$ to 2 , and $M$ consists of $r-1$ occurrences of 1 and $r$ occurrences of 2 .

Then $I^{\prime}$ is our resulting instance of \#XMGM, and we let $\mathcal{S}_{I^{\prime}}$ be its set of solutions. Notice that by definition of $\chi$ and $M, \mathcal{S}_{I^{\prime}}$ is the set of vdst $\left(T, \phi_{V}, \phi_{E}\right)$ of $H$ containing $r-1$ vertices mapped to $A$ and $r$ vertices mapped to $B$. We now show that we have a parsimonious reduction, by describing a bijection $\Phi$ : $\mathcal{S}_{I} \rightarrow \mathcal{S}_{I^{\prime}}$. Consider $\mathcal{T}=\left(T, \psi_{V}, \psi_{E}\right)$ in $\mathcal{S}_{I}$; we define $\Phi(\mathcal{T})=\left(T^{\prime}, \phi_{V}, \phi_{E}\right)$ as follows: 
- For each edge $e=u v \in E(T)$, we have $f_{e}:=\psi_{E}(e) \in E(G)$ : we then subdivide $e$, creating a new vertex $x_{e}$. Let $T^{\prime}$ be the resulting tree;

- For each vertex $x_{e}$, we define $\phi_{V}\left(x_{e}\right)=a\left[f_{e}\right]$. For each other vertex $u$ of $T^{\prime}$, we have $u \in V(T)$, let $(v, i)=\psi_{V}(u)$; we then set $\phi_{V}(u)=b[v, i]$ (this is possible since if $v \in V_{j}$ then $1 \leq i \leq t_{j}$, by definition of $\psi_{V}$ ).

From $\phi_{V}$, we then define $\phi_{E}$ in a natural way. Then $\mathcal{T}^{\prime}=\Phi(\mathcal{T})$ is indeed in $\mathcal{S}_{I^{\prime}}$ : (i) $\mathcal{T}^{\prime}$ is a vertex distinct subtree of $H$ (by definition of $\phi_{V}$ and since $\mathcal{T}$ was edgedistinct, the values $\phi_{V}\left(x_{e}\right)$ are distinct; by injectivity of $\psi_{V}$, the other values $\phi_{V}(u)$ are distinct); (ii) it has $r-1$ vertices mapped to $A$ and $r$ vertices mapped to $B$. To prove that $\Phi$ is a bijection, we describe the inverse correspondence $\Psi: \mathcal{S}_{I^{\prime}} \rightarrow \mathcal{S}_{I}$. Consider $\mathcal{T}^{\prime}=\left(T^{\prime}, \phi_{V}, \phi_{E}\right)$ in $\mathcal{S}_{I^{\prime}} ;$ we define $\Psi\left(\mathcal{T}^{\prime}\right)=\left(T, \psi_{V}, \psi_{E}\right)$ as follows. Let $A^{\prime}, B^{\prime}$ be the vertices of $T^{\prime}$ mapped to $A, B$ respectively. Let $i$ be the number of nodes of $A^{\prime}$ which are leaves: since the nodes of $A^{\prime}$ have degree 1 or 2 in $T^{\prime}$ depending on whether they are leaves or internal nodes, we then have $\left|E\left(T^{\prime}\right)\right| \leq i+2(r-1-i)=2 r-i-2$; since $\left|E\left(T^{\prime}\right)\right|=2 r-2$, we must have $i=0$. It follows that all leaves of $T^{\prime}$ belong to $B^{\prime}$; from $T^{\prime}$, by contracting each vertex of $A^{\prime}$ in $T^{\prime}$ we obtain a tree $T$ with $r$ vertices. We then define $\psi_{V}, \psi_{E}$ as follows: (i) given $u \in B^{\prime}$, if $\phi_{V}(u)=b[v, j]$, then $\psi_{V}(u)=(v, j$ ); (ii) given $e=u v \in E(T)$, there corresponds two edges $u x, v x \in E\left(T^{\prime}\right)$ with $x \in A^{\prime}$, and we thus have $\phi_{V}(x)=a[f]$, from which we define $\psi_{E}(e)=f$. It is easily seen that the resulting $\mathcal{T}=\Psi\left(\mathcal{T}^{\prime}\right)$ is in $\mathcal{S}_{I}$, and that the operations $\Phi$ and $\Psi$ are inverse of each other.

\section{Conclusion}

In this paper, we have obtained improved FPT algorithms for several variants of the Graph Motif problem. Reducing to the Multilinear Detection problem resulted in faster running times and a polynomial space complexity. We have also considered the counting versions of these problems, for the first time in the literature. Our results demonstrate that the algebraic framework of [16] has potential applications to computational biology, though a practical evaluation of the algorithms remains to be done. In particular, how do they compare to implementations based on color-coding or ILPs [8]

We conclude with some open questions. A first question concerns our results of Section 3.2 for multiset motifs: is it possible to further reduce the $\mathcal{O}^{*}\left(4^{k}\right)$ running times? Another question relates to the edge-weighted problems considered in Section 3.3. our algorithms are only pseudopolynomial in the maximum weight $r$, can this dependence in $r$ be improved? Finally, is approximate counting possible for the \#XMGM problem? We believe that some of these questions may be solved through an extension of the algebraic framework of Koutis and Williams. 


\section{References}

1. E. Alm and A.P. Arkin. Biological networks. Curr. Opin. Struct. Biol., 13(2):193202, 2003.

2. N. Alon, R. Yuster, and U. Zwick. Color-coding. J. of ACM, 42(4):844-856, 1995.

3. N. Betzler, M.R. Fellows, C. Komusiewicz, and R. Niedermeier. Parameterized Algorithms and Hardness Results for Some Graph Motif Problems. In CPM 2008, volume 5029 of $L N C S$, pages 31-43, 2008.

4. A. Björklund, T. Husfeldt, P. Kaski, and M. Koivisto. Fourier meets möbius: fast subset convolution. In STOC, pages 67-74, 2007.

5. G. Blin, F. Sikora, and S. Vialette. GraMoFoNe: a Cytoscape plugin for querying motifs without topology in Protein-Protein Interactions networks. In BICoB 2010, pages $38-43,2010$.

6. S. Böcker, F. Rasche, and T. Steijger. Annotating Fragmentation Patterns. In WABI 2009, volume 5724 of $L N B I$, pages 13-24, 2009.

7. S. Bruckner, F. Hüffner, R.M. Karp, R. Shamir, and R. Sharan. Topology-Free Querying of Protein Interaction Networks. In RECOMB, volume 5541 of $L N C S$, pages 74-89, 2009.

8. R. Dondi, G. Fertin, and S. Vialette. Weak pattern matching in colored graphs: Minimizing the number of connected components. In ICTCS, pages 27-38, 2007.

9. R. Dondi, G. Fertin, and S. Vialette. Maximum Motif Problem in Vertex-Colored Graphs. In CPM 2009, volume 5577 of $L N C S$, pages 221-235, 2009.

10. M.R. Fellows, G. Fertin, D. Hermelin, and S. Vialette. Sharp Tractability Borderlines for Finding Connected Motifs in Vertex-Colored Graphs. In ICALP 200\%, volume 4596 of $L N C S$, pages 340-351, 2007.

11. J. Flum and M. Grohe. The Parameterized Complexity of Counting Problems. SIAM Journal on Computing, 33(4):892-922, 2004.

12. J. Flum and M. Grohe. Parameterized Complexity Theory. Springer-Verlag, 2006.

13. F. Hüffner, S. Wernicke, and T. Zichner. Algorithm Engineering For Color-Coding To Facilitate Signaling Pathway Detection. In APBC 2007, pages 277-286, 2007.

14. R.M. Karp. Dynamic-programming meets the principle of inclusion and exclusion. Oper. Res. Lett., 1:49-51, 1982.

15. I. Koutis. Faster Algebraic Algorithms for Path and Packing Problems. In ICALP 2008, volume 5125 of LNCS, pages 575-586, 2008.

16. I. Koutis and R. Williams. Limits and Applications of Group Algebras for Parameterized Problems. In ICALP, volume 5555 of $L N C S$, pages 653-664, 2009.

17. V. Lacroix, C.G. Fernandes, and M.-F. Sagot. Motif Search in Graphs: Application to Metabolic Networks. Trans. Comput. Biol. Bioinform., 3(4):360-368, 2006.

18. J. Nederlof. Fast Polynomial-Space Algorithms Using Möbius Inversion: Improving on Steiner Tree and Related Problems. In ICALP 2009, volume 5555 of LNCS, pages 713-725, 2009.

19. S. Schbath, V. Lacroix, and M.-F. Sagot. Assessing the exceptionality of coloured motifs in networks. EURASIP JBSB, pages 1-9, 2009.

20. R. Sharan and T. Ideker. Modeling cellular machinery through biological network comparison. Nature Biotechnology, 24:427-433, 2006.

21. R. Williams. Finding paths of length $\mathrm{k}$ in $O^{*}\left(2^{k}\right)$ time. $I P L, 109(6): 315-318,2009$. 


\section{Appendix}

\subsection{End of proof of Proposition 1}

Given a set $S \subseteq C$, define the multilinear monomial $\pi_{S}:=\prod_{c \in S} x_{c}$. Given $u \in V(T)$ and $S \subseteq C$, an $(u, S)$-solution is a subtree $T=\left(V_{T}, E_{T}\right)$ of $G$, such that $u \in V_{T}, T$ is distinctly colored by $\chi$, and $\chi\left(V_{T}\right)=S$. We show by induction on $1 \leq i \leq k$ that: $\pi_{S}$ is a multilinear monomial of $P_{i, u}$ iff (i) $|S|=i$ and (ii) there exists an $(u, S)$-solution. This is clear when $i=1$; now, suppose that $i \geq 2$, and assume that the property holds for every $1 \leq j<i$.

Suppose that $|S|=i$ and that $T=\left(V_{T}, E_{T}\right)$ is an $(u, S)$-solution, let us show that $\pi_{S}$ is a multilinear monomial of $P_{i, u}$. Let $v$ be a neighbor of $u$ in $T$, then removing the edge $u v$ from $T$ produces two trees $T_{1}, T_{2}$ with $T_{1}$ containing $u$ and $T_{2}$ containing $v$. These two trees are distinctly colored, let $S_{1}, S_{2}$ be their respective color sets, and let $i_{1}, i_{2}$ be their respective sizes. Since $T_{1}$ is an $\left(u, S_{1}\right)$-solution, $\pi_{S_{1}}$ is a multilinear monomial of $P_{i_{1}, u}$ by induction hypothesis. Since $T_{2}$ is a $\left(v, S_{2}\right)$-solution, $\pi_{S_{2}}$ is a multilinear monomial of $P_{i_{2}, v}$ by induction hypothesis. It follows that $\pi_{S}=\pi_{S_{1}} \pi_{S_{2}}$ is a multilinear monomial of $P_{i_{1}, u} P_{i_{2}, v}$, and thus of $P_{i, u}$.

Conversely, suppose that $\pi_{S}$ is a multilinear monomial of $P_{i, u}$. By definition of $P_{i, u}$, there exists $1 \leq i^{\prime} \leq i-1$ and $v \in N_{G}(u)$ such that $\pi_{S}$ is a multilinear monomial of $P_{i^{\prime}, u} P_{i-i^{\prime}, v}$. We can then partition $S$ into $S_{1}, S_{2}$, with $\pi_{S_{1}}$ multilinear monomial of $P_{i^{\prime}, u}$ and $\pi_{S_{2}}$ multilinear monomial of $P_{i-i^{\prime}, v}$. Induction hypothesis therefore implies that (i) $\left|S_{1}\right|=i^{\prime}$ and $\left|S_{2}\right|=i-i^{\prime}$, (ii) there exists an $\left(u, S_{1}\right)$-solution $T_{1}=\left(V_{1}, E_{1}\right)$ and a $\left(v, S_{2}\right)$-solution $T_{2}=\left(V_{2}, E_{2}\right)$. Since $S_{1}, S_{2}$ are disjoint, it follows that $|S|=i$, which proves (i); besides, $V_{1}, V_{2}$ are disjoint, and thus $T=\left(V_{1} \cup V_{2}, E_{1} \cup E_{2} \cup\{u v\}\right)$ is an $(u, S)$-solution, which proves (ii).

\subsection{Proof of Lemma 1}

We first reduce \#Multicolored Clique to \#MEST - 1. Our source problem \#Multicolored Clique is the counting version of Multicolored Clique, which is easily seen to be \#W[1]-hard. Let $I=(G, k)$ be an instance of the problem, where $G=(V, E)$ has a partition $\mathcal{P}$ into classes $V_{1}, \ldots, V_{k}$. Our target instance is $I^{\prime}=(k, r, H, t)$ with $r=k^{2}-k+1$ and $t=(k, k-1, \ldots, k-1)$. The graph $H$ is obtained by splitting every edge $e$ in two parallel edges; then $H$ is a $k$-partite multigraph with partition $\mathcal{P}$. Let $\mathcal{S}_{I}, \mathcal{S}_{I^{\prime}}$ be the solution sets of $I$ and $I^{\prime}$ respectively. Let $\mathcal{K}_{k}$ be the multigraph with $k$ vertices $1, \ldots, k$, and with two parallel edges between distinct vertices; its partition is $\mathcal{P}_{k}$ consisting of the sets $\{1\}, \ldots,\{k\}$. Let $\mathcal{U}_{k}$ denote the set of $\left(\mathcal{P}_{k}, t\right)$-labeled edsts $\left(\mathcal{T}, \psi_{V}, \psi_{E}\right)$ for $\mathcal{K}_{k}$ such that $T\left(\psi_{V}\right)=t$. Observe that $\mathcal{U}_{k} \neq \emptyset$ : since every vertex of $\mathcal{K}_{k}$ has degree $2(k-1)$, it follows that $\mathcal{K}_{k}$ has an Eulerian path starting at 1 , which visits $k$ times the vertex 1 , and each other vertex $k-1$ times. We claim that $\left|\mathcal{S}_{I^{\prime}}\right|=\left|\mathcal{U}_{k}\right|\left|\mathcal{S}_{I}\right|$, which will prove the correctness of the reduction. To this aim, we will describe a bijection $\Phi: \mathcal{S}_{I^{\prime}} \times \mathcal{U}_{k} \rightarrow \mathcal{S}_{I}$. 
Consider a pair $P=(C, \mathcal{T}) \in \mathcal{S}_{I^{\prime}} \times \mathcal{U}_{k}$ with $\mathcal{T}=\left(T, \psi_{V}, \psi_{E}\right)$ and $C=$ $\left\{v_{1}, \ldots, v_{k}\right\}$ multicolored clique of $G$ (with $\left.v_{i} \in V_{i}\right)$. Let $\phi=\left(\phi_{V}, \phi_{E}\right)$ be the homomorphism of $\mathcal{K}_{k}$ into $H$ which maps $i$ to $v_{i}$, and the parallel edges accordingly. We then define $\mathcal{T}^{\prime}=\Phi(P)$ by $\mathcal{T}^{\prime}=\left(T, \psi_{V}^{\prime}, \psi_{E}^{\prime}\right)$, where (i) $\psi_{V}^{\prime}$ is defined so that if $\psi_{V}(u)=(v, i)$ and if $\phi_{V}(v)=w$ then $\psi_{V}^{\prime}(u)=(w, i)$, (ii) $\psi_{E}^{\prime}=\psi_{E} \circ \phi_{E}$. We verify that $\mathcal{T}^{\prime} \in \mathcal{S}_{I}$ : indeed, it is a $(\mathcal{P}, t)$-labeled edst of $G$ and $T\left(\psi_{V}^{\prime}\right)=t$ (since we have composed with injective functions $\phi_{V}, \phi_{E}$ ). To prove that $\Phi$ is a bijection, we define the inverse function $\Psi: \mathcal{S}_{I} \rightarrow \mathcal{S}_{I^{\prime}} \times \mathcal{U}_{k}$ as follows. Consider $\mathcal{T}^{\prime}=\left(T, \psi_{V}^{\prime}, \psi_{E}^{\prime}\right)(\mathcal{P}, t)$-labeled edst of $G$, with $T\left(\psi_{V}^{\prime}\right)=t$. This equality yields vertices $v_{1} \in V_{1}, \ldots, v_{k} \in V_{k}$ such that $\left|\left(\psi_{V}^{r}\right)^{-1}\left(v_{i}\right)\right|=t_{i}$. Let $C=\left\{v_{1}, \ldots, v_{k}\right\}$, then $C$ is a multicolored clique of $G$ : indeed, $H[C]$ has at most $k^{2}-k$ edges, and since $\psi_{E}^{\prime}$ is injective it must have exactly $k^{2}-k$ edges, implying that $G[C]$ is a complete graph. We can then define $\left(\psi_{V}, \psi_{E}\right)$ from $\left(\psi_{V}^{\prime}, \psi_{E}^{\prime}\right)$ by "projecting" $v_{i}$ on $i$, and the parallel edges accordingly (for instance, if $\psi_{V}^{\prime}(u)=\left(v_{i}, j\right)$ then $\left.\psi_{V}(u)=(i, j)\right)$. We finally define $P=\Psi\left(\mathcal{T}^{\prime}\right)$ by $P=(C, \mathcal{T})$ where $\mathcal{T}=\left(T, \psi_{V}, \psi_{E}\right)$. It is easy to see that $P \in \mathcal{S}_{I^{\prime}} \times \mathcal{U}_{k}$, and that $\Phi$ and $\Psi$ are inverse of each other.

We now give a Turing-reduction of \#MEST -1 to \#MEST -2 . Given a tuple $t \in[r]^{k}$, we define the instance $I_{t}=(k, r, G, t)$, and we let $\mathcal{S}_{t}, \mathcal{S}_{t}^{\prime}$ be its solution sets for \#MEST - 1, \#MEST -2 respectively. Let $N_{t}=\left|\mathcal{S}_{t}\right|$ and $N_{t}^{\prime}=\left|\mathcal{S}_{t}^{\prime}\right|$. We have for every $t \in[r]^{k}: N_{t}^{\prime}=\sum_{t^{\prime} \leq t} N_{t^{\prime}}$, which yields by Möbius inversion that for every $t \in[r]^{k}: N_{t}=\sum_{t^{\prime}<t} \mu\left(t, t^{\prime}\right) N_{t^{\prime}}^{\prime}$. Therefore, we can compute a value $N_{t}$ using $\mathcal{O}\left(2^{k}\right)$ oracle calls for \#MEST -2 , thereby solving \#MEST -1 .

\footnotetext{
${ }^{5}$ where $\mu\left(t, t^{\prime}\right)$ is 0 if there exists $i \in[k]$ s.t. $t_{i}-t_{i}^{\prime}>1$, and is otherwise equal to $(-1)^{r}$ where $r$ is the number of $i \in[k]$ s.t. $t_{i}-t_{i}^{\prime}=1$.
} 